\title{
Fabricación de la Aleación Au-Si para Utilización como Soldadura Blanda de Aleaciones de Oro
}

\author{
(Fabrication of Au-Si Alloys for Using in Soldering of Gold Alloys)
}

\author{
Julián Tobón ${ }^{1}$, Claudia P. Serna-Giraldo ${ }^{2 *}$, Héctor Sánchez ${ }^{2}$ \\ ${ }^{1}$ Magister en Ingeniería, Universidad de Antioquia, Medellin, Antioquia, Colombia,juliusness@gmail.com \\ ${ }^{2}$ Docentes del Departamento de Ingeniería Metalúrgica y de Materiales, Universidad de Antioquia. Investigadores grupo GIPIM- \\ ME,Medellín,Antioquia,Colombia, claserna@udea.edu.co,hsanchez@udea.edu.co
}

\begin{abstract}
Resumen
La Aleación Au-Si en su composición eutéctica presenta un punto de fusión cercano a los $369^{\circ} \mathrm{C}$ y color similar al oro de alta ley. En este estudio se presentan resultados de fabricación de las aleaciones de oro con 2,5; 2,8 y 3,0\% de silicio, por medio de la fusión en un horno de plasma con atmósfera inerte de argón. Se realizó caracterización de composición química por espectroscopía de dispersión de energía (EDS) y espectrometría de dispersión de longitud de onda (WDS), análisis metalográfico y microestructural por microscopía óptica, SEM y DRX, dureza y microdureza Vickers, evaluación del punto de fusión por DSC y determinación de las coordenadas colorimétricas SCIELab. A nivel microestructural las aleaciones muestran pequeñas formaciones dendríticas ricas en oro y el constituyente eutéctico formado por precipitados de silicio aciculares en una matriz de oro. Los resultados de dureza y microdureza dieron valores alrededor de los $110 \mathrm{HV}$, presentándose un importante aumento en las propiedades mecánicas con respecto a las aleaciones tradicionales de oro de alta pureza. La temperatura de fusión para las aleaciones se ubicó en los $374^{\circ} \mathrm{C}$, además de presentar un color muy cercano a los valores del oro puro.
\end{abstract}

Palabras claves: Aleaciones de Oro. Aleaciones Au-Si. Oro de Alta Ley. Soldadura Blanda de Oro.

Resumo: A liga Au-Si na composição eutéctica tem o ponto de fusão perto dos $369^{\circ} \mathrm{C}$ e tem a cor similar ao ouro puro, de alta kilatagem. Essa característica pode ser aproveitada no campo da joalharia e da eletrónica, para ser utilizado como um material de aporte de soldagem branda. Nesta pesquisa apresentam-se resultados de fabricação de ligas de ouro com 2,5; 2,8 e 3,0\% de Silicio. A fusão foi feita num forno de plasma com atmósfera de gas argônio. A caracterização foi feita mediante ensaios de composição química no EDS e WDS acoplado ao MEV, análise metalográfica e microestrutural mediante microscopia óptica, microscopia electrónica de varredura, difração de raios $X$, dureza e microdureza Vickers, análise de ponto de fusão por calorimetria diferencial de varredura e a determinação de coordenadas de cor pelo método SCIELab. Na microestructura, as ligas mostraram pequenas formações dendríticas enriquecidas em ouro e o constituinte eutéctico está formado por precipitados aciculares de silício numa matriz de ouro. Os resultados de dureza e microdureza mostraram valores ao redor dos $110 \mathrm{HV}$. Apresentou-se um aumento importante nas propriedades mecánicas respeito às ligas tradicionais de ouro de alta pureza. A temperatura de fusão das ligas foi de $374^{\circ} \mathrm{C}$, além de que a cor se manteve muito próxima ao ouro puro.

Palavras chaves: Ligas de Ouro. ligas Au-Si., Ouro Alta Kilatagem. Soldagem Branda de Ouro.

\begin{abstract}
The Au-Si alloys have a melting temperature at $369^{\circ} \mathrm{C}$ in the eutectic composition, and the color is similar to the high purity. These characteristics can be exploited in the field of jewelry and electronics for used as filler soldering. In this research, are showing results of manufacturing Au-Si alloys with composition 2,2\% Si, 2,8\% Si and 3,0\% Si. The fusion was made in the plasma furnace whit inert argon protection, and the characterization was made by chemical composition using the EDS of Scanning Electronic Microscope (SEM). The microstuctural analysis was made by optical microscope, SEM, DRX, Vickers hardness and microhardness. The melting temperature was evaluates by Diferencial Scanning Calorimetry (DSC), the color measure was made by CIELab. The microstructure alloys showed dendritics Au phase and the eutectic constituent were silicon acicular precipitates in the gold matrix. The hardness and microhardness values were near $110 \mathrm{HV}$, and so, the properties were better than high purity gold alloys. The melting temperature were near the $374^{\circ} \mathrm{C}$, also showed a color very similar to the high purity gold alloys.
\end{abstract}

Key words: Gold alloys. Au-Si Alloys. High Purity Golden Alloys. Gold Soldering.

Recebido em 02/10/2012. Texto Final em 02/07/2013.

*Autor para quem toda a correspondência debe ser encaminhada: Claudia Patricia Serna-Giraldo, claserna@udea.edu.co 


\section{Introducción}

El oro $(\mathrm{Au})$ es un metal precioso que en su estado puro se caracteriza por tener alta ductilidad, baja dureza y baja resistencia a la tracción. Con el fin de mejorar sus propiedades mecánicas y a la vez el desempeño en sus diferentes campos de aplicación, es necesario alearlo con otros elementos, mejorando así la resistencia a la tracción y aumentando los valores de dureza. En el campo de la joyería además de las propiedades mecánicas, el color es una propiedad muy importante que varía de acuerdo a la ley de la aleación (la cual indica el grado de pureza del oro) y nominalmente se definen como 10, 14, 18 o 24 quilates [1].

Las aleaciones base oro con menos de $2 \%$ de aleantes se han denominado aleaciones de alta ley. Estas aleaciones han tenido aplicaciones limitadas por las bajas propiedades mecánicas. No obstante, se han realizado algunas investigaciones para mantener la alta ley de oro, usando como aleante el titanio, el cual permite mejorar las propiedades mecánicas y a su vez conservar el color amarillo característico del oro puro [2-4]. En este tipo de aleaciones, una de las alternativas para la fabricación de joyas mas económicas es por medio de láminas delgadas conformadas $\mathrm{y}$ unidas por procesos de soldadura blanda, lo cual exige el uso de material de aporte que conserve las características de alta ley del sustrato. Se han realizado algunos estudios sobre diferentes aleaciones para usarse como material de aporte para este propósito [5]. Este material de aporte deberá cumplir con diferentes requerimientos, el principal es tener un bajo punto de fusión, por debajo de $450^{\circ} \mathrm{C}$, para evitar la modificación de la microestructura del sustrato que puede deteriorar las propiedades mecánicas.

En general, las soldaduras de oro que han sido usadas para joyería, electrónica, energía nuclear y tecnología aeroespacial han sido basadas en el sistema oro-cobre y oro-níquel, las cuales presentan buenas características de resistencia a la corrosión y resistencia mecánica, y presentan un punto de fusión que supera los $900^{\circ} \mathrm{C}$ y además que no requiere de fundentes para su aplicación [6]. Metales de aporte con punto de fusión inferior a $450^{\circ} \mathrm{C}$ han sido propuestos por Humpston [7], permitiendo la unión de aleaciones de oro de alta ley de 22 quilates con aleaciones constituidas de oro-silicio y oro-germanio, las cuales presentan un color dorado muy similar al oro puro, pues otras aleaciones como oro-estaño y oro-antimonio, a pesar de presentar puntos de fusión menores de $360^{\circ} \mathrm{C}$, presentan un color blanco-grisáceo. Una buena alternativa, son las aleaciones Au-Si con composiciones próximas al eutéctico las cuales presentan propiedades que la hacen apta para aplicaciones como metal de aporte en oro de alta ley. Particularmente el bajo punto de fusión, fluidez, acción capilar (coeficiente de esparcido y mojabilidad), adherencia y el color amarillo similar al sustrato, son las características que la hacen más atractiva [5]. La influencia del silicio en las aleaciones de oro depende de su concentración y de la composición de la aleación, y se debe asumir que una óptima concentración de silicio debe desarrollarse para cada aplicación particular. De un lado, se reporta que adiciones de silicio cercanas a $0,25 \%$ en oro fundido de 9 quilates mejora la ductilidad [8]. De otro lado, se reporta el efecto fragilizante del silicio en oro de 18 quilates fundido, mejorando sus propiedades con adiciones de níquel y de cobalto [9]. No se han encontrado trabajos que reporten el comportamiento de la aleación Au-Si con composición próximas al eutéctico para ser utilizados como material de aporte en soldaduras blandas de oro de alta ley.

En la Figura 1 se muestra el diagrama de fase Oro-Silicio [5], en el que se puede ver como el Silicio no presenta solubilidad en el oro además de bajar la temperatura de fusión de este, hasta $369^{\circ} \mathrm{C}$, en el punto eutéctico con una composición de $3,16 \% \mathrm{Si}$. Esta es una característica que lo hace adecuado como metal de aporte para soldadura blanda de oro.

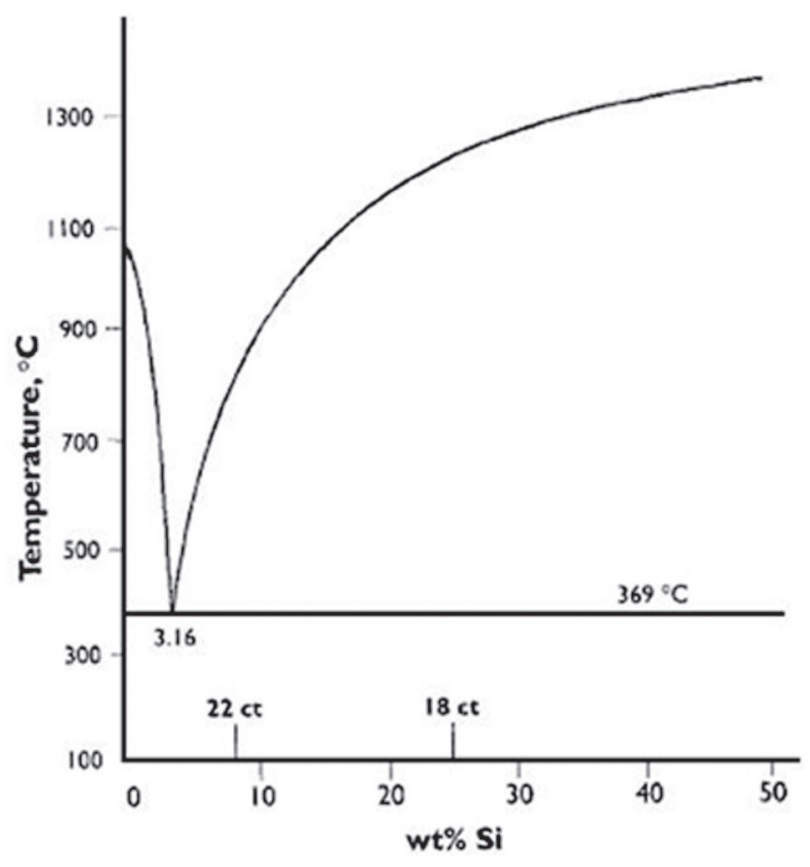

Figura 1. Diagrama de fase Au-Si [5]

El objetivo de este trabajo es fabricar y caracterizar tres aleaciones de oro con porcentajes nominales de silicio próximos al eutéctico en composiciones de 2,5; 2,8 y 3,0 \%, para posteriormente evaluar su posibilidad de uso como material de aporte en soldadura blanda de aleaciones de oro de alta ley.

\section{Metodología}

Para la fabricación de las aleaciones Au-Si fue necesario partir de oro y silicio puro. Para obtener oro puro se partió de oro comercial de 18 quilates el cual se sometió a un proceso de refinación por el método del ácido nítrico [10]. En este método se funde el oro de 18 quilates con cobre electrolítico, produciendo una aleación con una relación 1:3 en peso de oro y cobre. Esta aleación es disuelta en una solución de ácido nítrico con temperatura controlada, esta disolución favorece la precipitación del oro puro. El silicio utilizado para la producción de las aleaciones fue triturado y separado por las partículas pasantes malla 10 (2 $\mathrm{mm})$ y retenidas malla $20(1 \mathrm{~mm})$. Tanto el oro como el silicio fueron caracterizados composicionalmente utilizando el ensayo EDS, además al oro se le realizó prueba de densidad relativa, que es un ensayo fácil de realizar para determinar de manera preliminar el grado de pureza del oro refinado. 

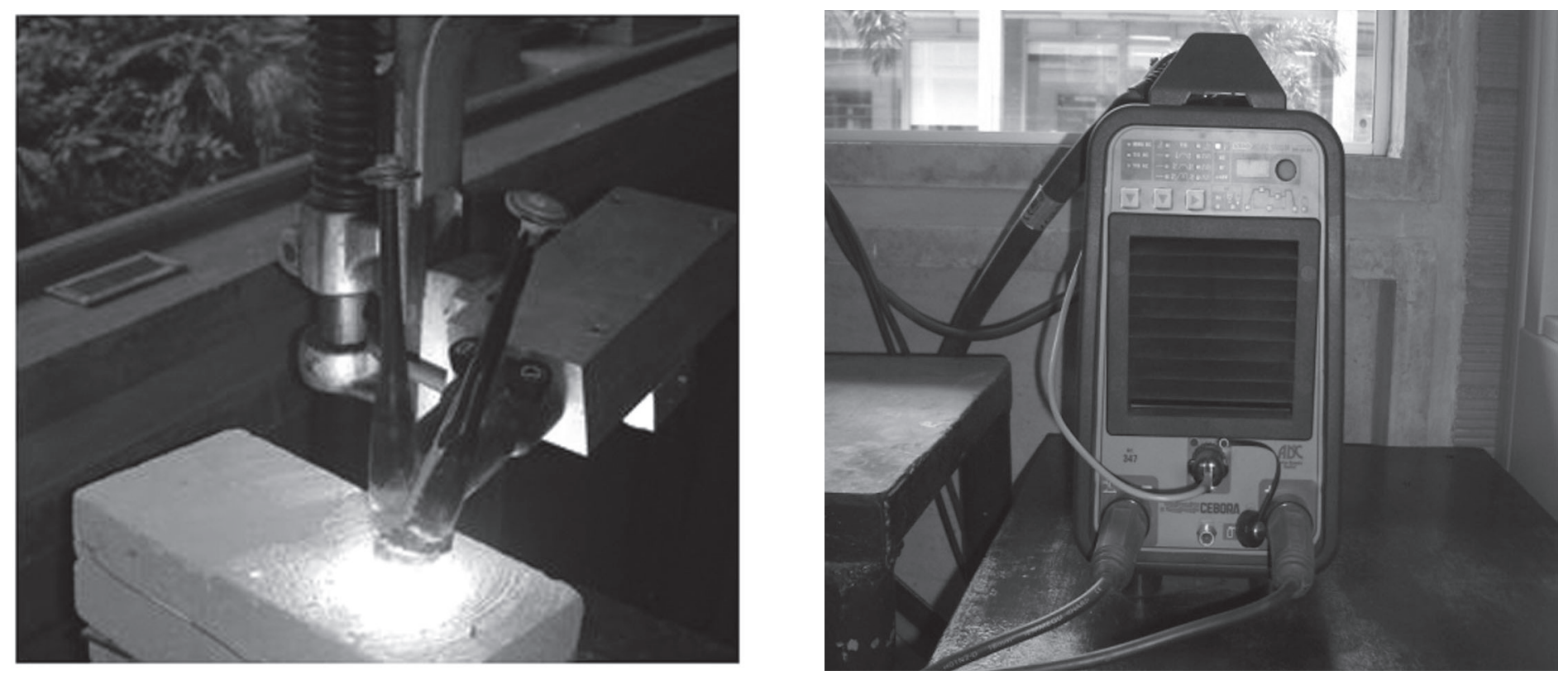

Figura 2. Horno de fusión por plasma

La fusión de las aleaciones Au-Si se realizó en un horno de fusión por plasma con atmósfera inerte de argón construido en la Universidad de Antioquia, el cual se muestra en la Figura 2. En esta etapa fue importante garantizar las mismas condiciones de calentamiento, temperatura de fusión, tiempo de permanencia y régimen de enfriamiento para la disolución del silicio en el oro. La atmósfera de argón, con un flujo de $50 \mathrm{CFH}$ (pies cúbicos por hora), permitió controlar las pérdidas de silicio durante la fusión. Este control se garantizó fijando los parámetros de operación como son precalentamiento de crisoles y ladrillos refractarios a $900^{\circ} \mathrm{C}$, corriente $\mathrm{AC}$ de 90 amperios y tiempo de permanencia, después de la fusión del oro, de tres minutos, para la disolución del silicio. Una vez disuelto el silicio se disminuyó la corriente a razón de 35 amperios por minuto. El metal fue fundido en crisoles de alúmina dentro de refractario aislante para concentrar el calor.

Se fabricaron tres aleaciones base oro con porcentajes nominales de silicio de 2,5; 2,8 y 3,0 los cuales están cercanos a la composición eutéctica conforme se observa del diagrama de fases de Au-Si que se muestra en la Figura 1.

A las aleaciones obtenidas se realizaron mediciones de densidad y análisis de composición química por el método EDS y WDS para confirmar el contenido de oro. Para la caracterización microestructural se realizó pulido convencional y acabado en paño con alúmina de $3 \mu \mathrm{m}$ y luego fueron atacadas con agua regia recién preparada, para ser observadas en microscopio óptico marca OLYMPUS modelo PME3 y en microscopio electrónico de barrido JEOL JSM-6490LV. El análisis microestructural fue complementado con ensayos DRX, para determinar las fases presentes. Se realizaron ensayos de dureza Vickers con carga de $3 \mathrm{Kg}$ en durómetro marca Härteprüfer Gnehm referencia Swiss Max 300. Se midió microdureza Vickers con carga de 100g, en un microdurómetro marca Shimadzu modelo NT M001. La temperatura de fusión de las aleaciones se determinó mediante calorimetría diferencial de barrido, utilizando un equipo DSC
Q200 V24.2 Build 107. Finalmente, se realizaron estudios de color por espectrometría óptica en un espectrómetro AVANTES AVAESPEC-2048, utilizando una fuente de luz halógena y una de deuterio. Para simular el comportamiento como metal de aporte en una soldadura de oro, las tres aleaciones producidas fueron laminadas hasta $0,1 \mathrm{~mm}$, y depositadas sobre un sustrato de Au-Ti previamente laminado de 0,3 mm de espesor. Se utilizó una antorcha de gas propano, que usualmente se utiliza en joyería para este propósito. Como fundente se utilizó una solución sobresaturada de agua y bórax al $60 \%$. Este depósito fue caracterizado microestructuralmente en la intercara. En investigaciones posteriores a esta se pretende evaluar la mojabilidad de las aleaciones Au-Si, mediante la medición del ángulo.

\section{Resultados y discusión}

Los análisis de composición química obtenidos por EDS y la densidad relativa del oro refinado, utilizados para la fabricación de las aleaciones se muestran en la Tabla 1. Estos resultados evidencian el nivel de pureza de las materias primas, el oro tiene una densidad relativa teórica de 19.3.

Tabla 1. Resultados de la caracterización de las materias primas

\begin{tabular}{|c|c|c|}
\hline Material & Densidad & Composición (\%w) \\
\hline $\mathrm{Au}$ & 19.25 & 100.00 \\
\hline
\end{tabular}

En la Figura 3 se muestran las micrografías obtenidas por microscopía óptica para las tres composiciones producidas. Se observa que la microestructura está compuesta por un constituyente principal conformado por una matriz de oro (fase clara) y precipitados de silicio (fase oscura). Se evidencia la presencia de dendritas, las cuales son constituidas principalmente por oro. 


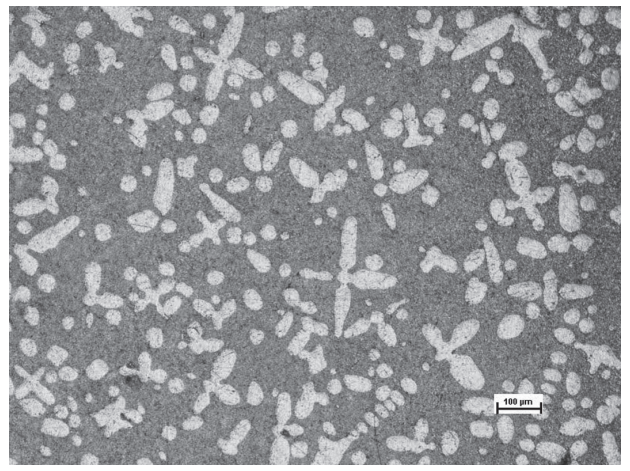

1)

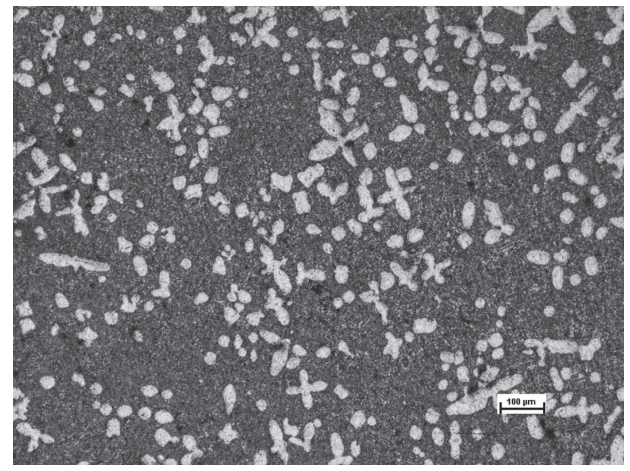

2)

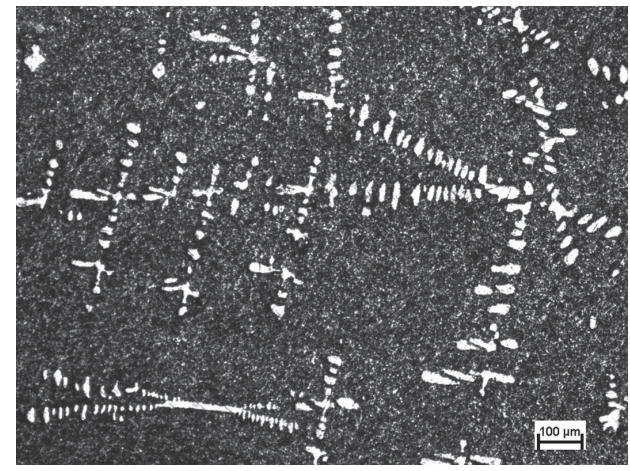

3)

Figura 3. Micrografías por microscopía óptica para las muestras a) $2,5 \% \mathrm{Si}$, b) $2,8 \% \mathrm{Si}$ y c) $3 \% \mathrm{Si}$.
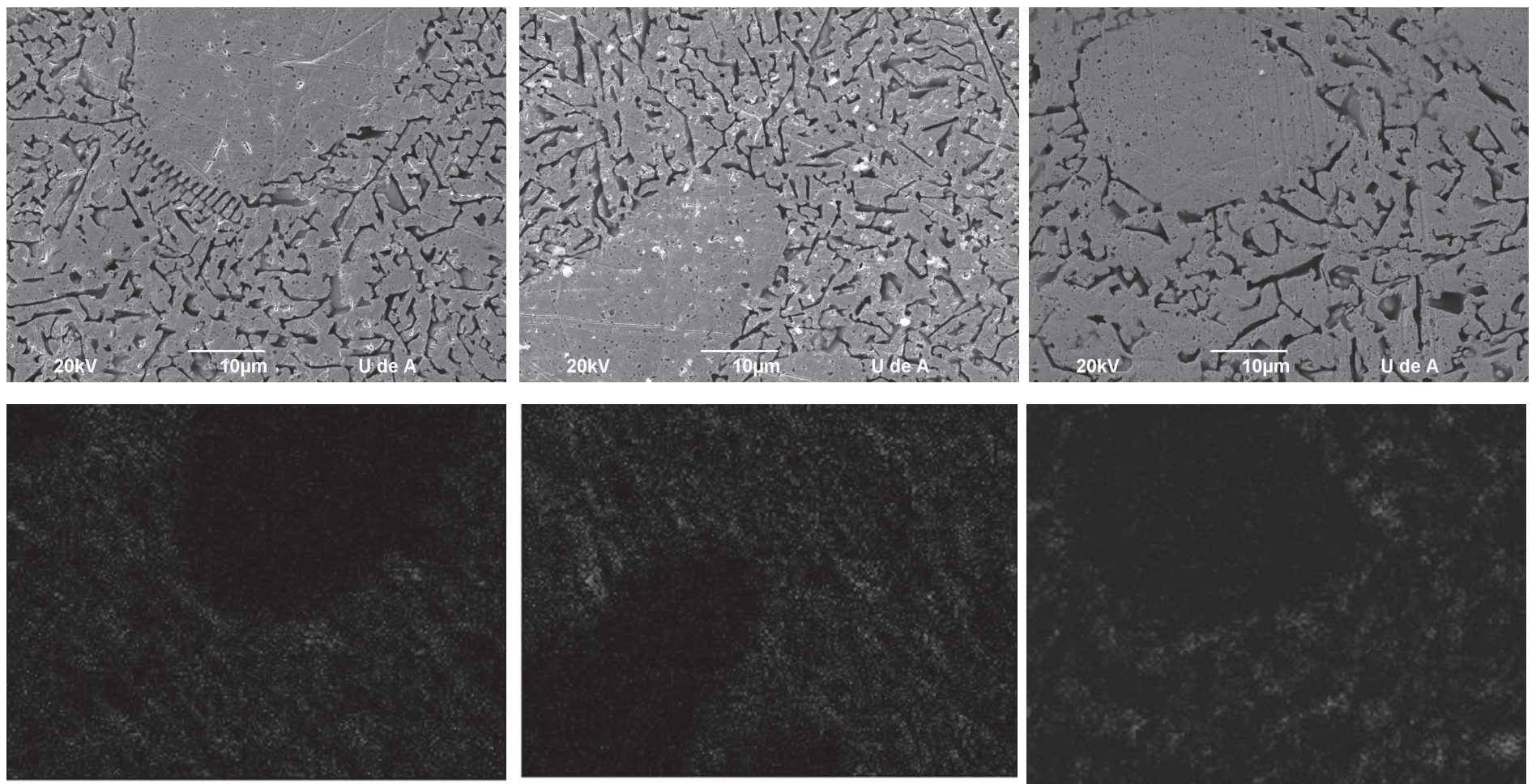

Si Ka1

1)

2)

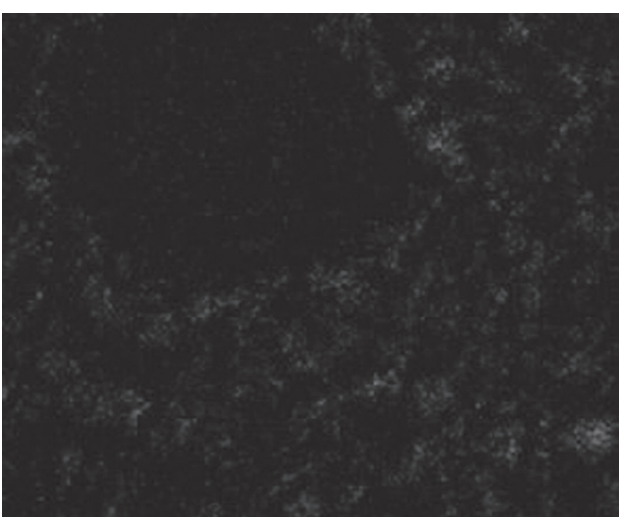

3)

Comparación de las micrografías SEM y de la distribución del silicio según las fases presentes a) y c) 2,5 \% $\mathrm{Si}$, b) y e) 2,8\%Si y c) y f) $3 \% \mathrm{Si}$

En la Figura 4 se muestran las imágenes obtenidos por SEM y el mapeo de composiciones realizado con EDS. En estas micrografías puede evidenciarse la morfología acicular del silicio. Además, se muestra la distribución del silicio, en color rojo, en las diferentes zonas de la aleación, evidenciando que éste se concentra en el constituyente eutéctico. En este mapeo de composición no se encontraron elementos contaminantes producto del proceso de fusión. En el análisis microestructural se hizo la medición de la variación del tamaño de las dendritas de oro con la disminución del porcentaje de silicio de las aleaciones, esta es presentada en la Figura 5, en la cual se muestra que a medida que se disminuye el contenido de silicio en las aleaciones se aumenta el tamaño de la dendrita de fase Au.

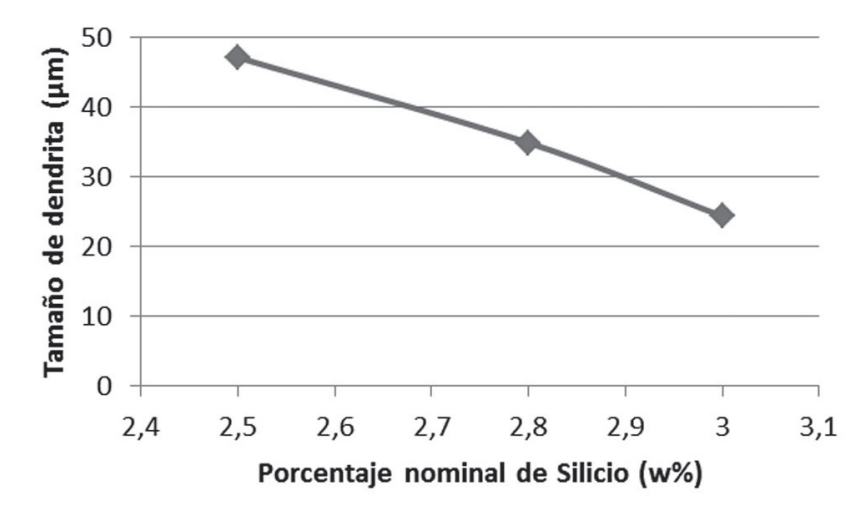

Figura 5. Tamaño de dendrita en función del porcentaje nominal de Silicio. 
Los resultados de DRX presentados en la Figura 6, mostraron que los picos de difracción corresponden a oro puro y silicio puro, lo que corrobora el hecho de que el silicio no presenta solubilidad en el oro y el oro tampoco es soluble en el silicio, ni forman compuestos intermetálicos.

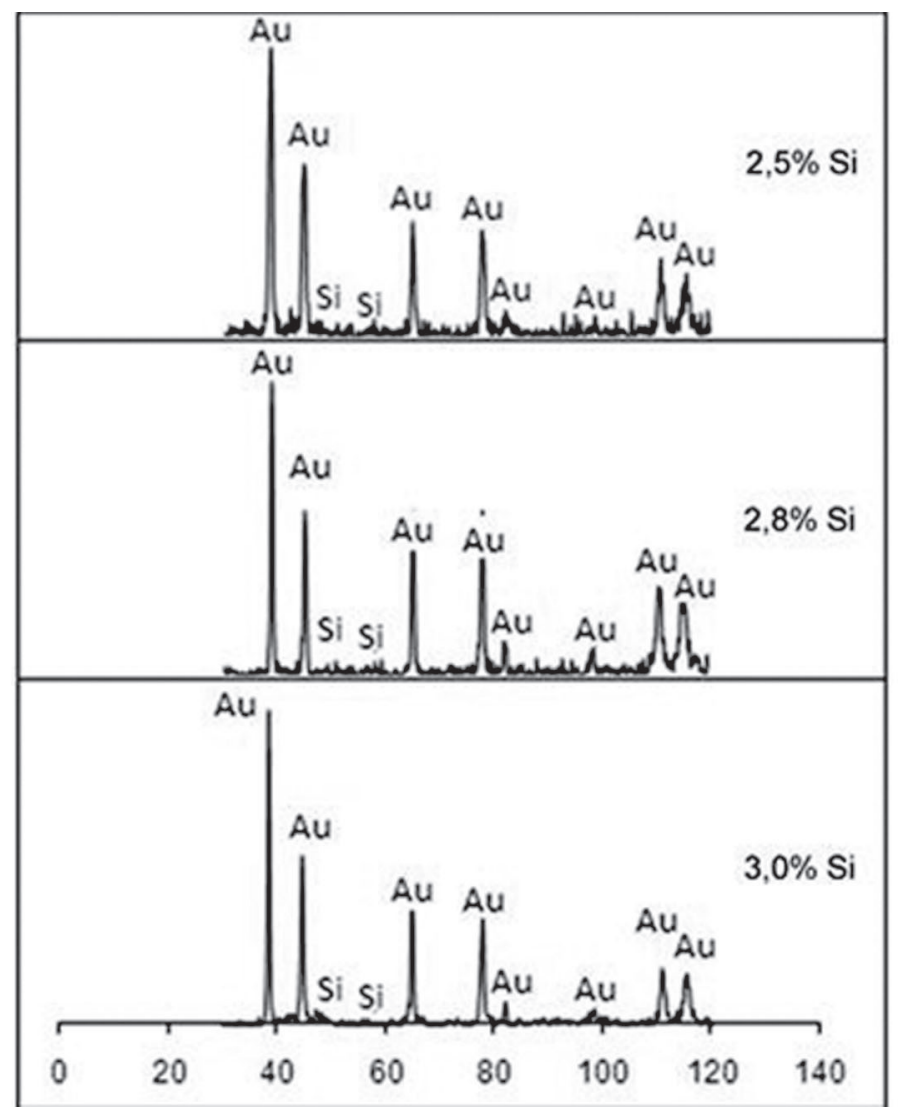

Figura 6. Espectros de difracción de rayos X para las tres muestras de Au-Si.

Análisis de composición en las dendritas y en el constituyente eutéctico se realizaron mediante espectrometría de dispersión de longitud de onda (WDS). Estos se representan en la Figura 7, en la cual se muestra como se distribuye el silicio. En la región dendrítica se pudo determinar presencia de silicio a medida que aumentaba el porcentaje nominal del mismo, debido a que el frente de solidificación de la fase oro, que no disuelve el silicio, alcanza a atrapar partículas. Además, estás mediciones permiten evidenciar que realmente en el constituyente eutéctico obtenido la concentración de silicio es mayor a la eutéctica de equilibrio, lo que puede ser consecuencia del proceso de enfriamiento después de la solidificación, el cual realmente no ocurrió en condiciones de equilibrio, pero tiene un régimen de enfriamiento lento, permitiendo que los precipitados de silicio en el eutéctico crecieran, aumentando la concentración de este. Además, a medida que aumenta el porcentaje de silicio en la aleación, este se precipita en las dendritas, disminuyendo su concentración en el eutéctico.

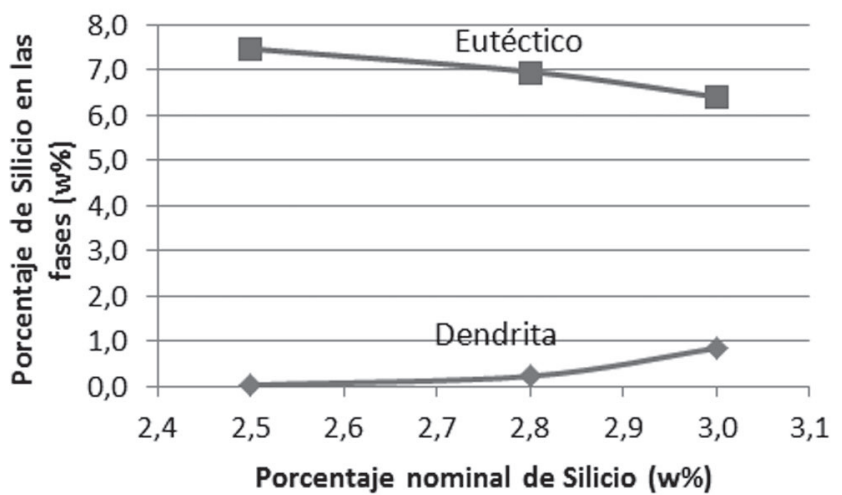

Figura 7. Variación del contenido de Silicio en la fase eutéctica y dendrítica según el porcentaje nominal de silicio

En la Figura 8 se muestra la variación de la densidad de la aleación con la variación del porcentaje nominal de silicio. Debe decirse en primer lugar, que hay una notable disminución en la densidad con respecto a la densidad del oro puro. Esto se explica por la baja densidad relativa del silicio que es de 2,33. Es importante aclarar que en estas aleaciones $\mathrm{Au}-\mathrm{Si}$, que se aproximan a alta ley por su alto tenor de $\mathrm{Au}$, ya no sería válido utilizar el criterio de densidad relativa para determinar la ley de la aleación. Pues como se tiene establecido, la ley de las aleaciones está determinada por el porcentaje de oro [1].

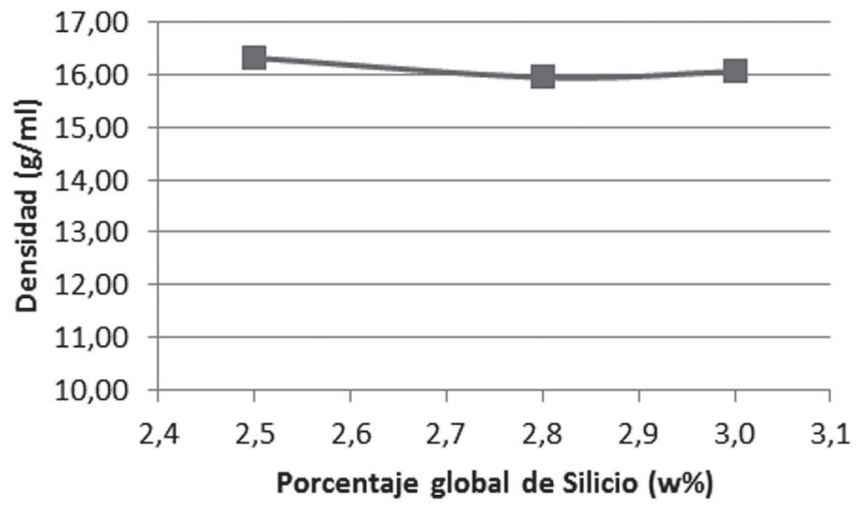

Figura 8. Densidad en función del porcentaje global de Silicio.

En la Figura 9 se presentan los resultados de microdureza tomados tanto para la fase dendrítica como para el constituyente eutéctico, de la cual se puede ver que en la fase dendrítica que tiene poco contenido de silicio es más blanda que la zona eutéctica. Si se considera por separado cada una de las fases de las diferentes aleaciones se observa que en la zona dendrítica se da un aumento en la microdureza debido al enriquecimiento en silicio como se mostró en la Figura 6. Como se dijo antes, el silicio no es disuelto por el oro pero los resultados muestran que se presenta un atrapamiento durante la solidificación de la fase dendrítica, generándose un aumento en el endurecimiento por la presencia de los precipitados de silicio. El reporte de la microdureza Vickers en la región eutéctica es afectado directamente por la distribución de los precipitados de silicio donde la 
microdureza mas alta se dio en la aleación con 2,8\% de silicio debió a que presenta una mejor distribución de los precipitados de silicio como se logra ver en la Figura 4. Los resultados de dureza Vickers presentados en la Tabla 2, muestran valores muy similares para las tres muestras de $\mathrm{Au}-\mathrm{Si}$, lo que muestra que a nivel global las aleaciones cercanas al eutéctico presentan poca variación, además estos valores de dureza son consistentes con los valores reportados de la microdureza.

Tabla 2. Resultados de dureza Vickers

\begin{tabular}{|c|c|c|}
\hline Aleación & Dureza Vickers & Desviación estándar \\
\hline $\mathrm{Au}-2,5 \% \mathrm{Si}$ & 103 & 4.64 \\
\hline $\mathrm{Au}-2,8 \% \mathrm{Si}$ & 105 & 5.69 \\
\hline $\mathrm{Au}-3,0 \% \mathrm{Si}$ & 104 & 4.69 \\
\hline
\end{tabular}

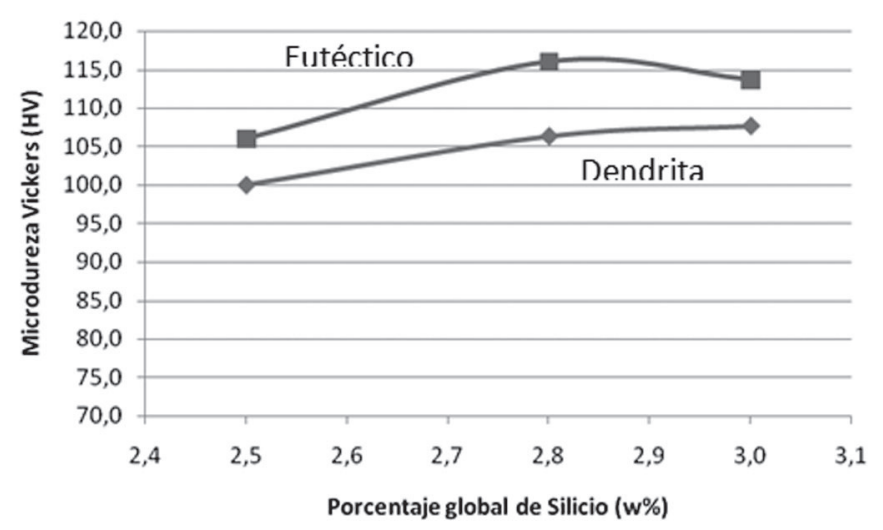

Figura 9. Microdureza en función del porcentaje global de Silicio.

Los ensayos DSC permitieron determinar las temperaturas de fusión de las aleaciones y sus entalpías de fusión, cuyos valores se muestran en la Tabla 3. Las temperaturas de fusión son muy similares para las tres aleaciones, alrededor de $\operatorname{los} 374^{\circ} \mathrm{C}$, muy inferior a $\operatorname{los} 450^{\circ} \mathrm{C}$ que corresponde al límite máximo de las soldaduras blandas, lo que permite corroborar que estas aleaciones son importantes candidatas para ser utilizadas como material de aporte en soldadura blanda. Las variaciones del punto de fusión con respecto al teórico se deben, como se dijo anteriormente, a que las aleaciones no fueron solidificadas en condiciones de equilibrio.

Tabla 3. Temperaturas de fusión y entalpías de fusión para las aleaciones Au-Si.

\begin{tabular}{|c|c|c|}
\hline Muestra & $\begin{array}{c}\text { Temperatura de } \\
\text { fusión }\left({ }^{\circ} \mathbf{C}\right)\end{array}$ & $\begin{array}{c}\text { Entalpía de fusión } \\
(\mathbf{J} / \mathbf{g})\end{array}$ \\
\hline $\mathrm{Au}-2,5 \% \mathrm{Si}$ & 374,08 & 42,52 \\
\hline $\mathrm{Au}-2,8 \% \mathrm{Si}$ & 374,31 & 42,56 \\
\hline $\mathrm{Au}-3,0 \% \mathrm{Si}$ & 373,29 & 53.83 \\
\hline
\end{tabular}

Para el ensayo de color, los resultados muestran una importante similitud de las aleaciones Au-Si con las aleaciones de 22 quilates $\mathrm{Au}-\mathrm{Ag}-\mathrm{Cu}$ y se acercan al color del oro puro. De la Figura 10 se puede apreciar la fuerte cercanía de la aleación Au$-3 \% \mathrm{Si}$ y una desviación hacia el color rojo de las muestras Au$-2,8 \% \mathrm{Si}$ y $\mathrm{Au}-2,5 \% \mathrm{Si}$, aunque los valores del Yellowness Index (YI), calculados según las normas ASTM D1925 y ASTM E313, permiten concluir que los valores de color se encuentran muy cercanos al color de las aleaciones de 22 quilates tradicionales. Los resultados de los índices de color YI se muestran en la Tabla 4 , el cual se constituye en una prueba importante en la medición del color en la industria de la joyería [9].

Tabla 4. Resultados de los indices de color YI para las muestras de Au-Si comparadas comparativamente con valores del oro puro y de aleaciones de 22 quilates tradicionales.

\begin{tabular}{|c|c|c|}
\hline Aleación & YI D1925 & YI E313 \\
\hline $\mathrm{Au}$ refinado & 70,46 & 53,93 \\
\hline $\mathrm{Au}-3 \% \mathrm{Si}$ & 63,71 & 48,67 \\
\hline $\mathrm{Au}-2,8 \% \mathrm{Si}$ & 61,89 & 48,06 \\
\hline $\mathrm{Au}-2,5 \% \mathrm{Si}$ & 63,71 & 48,67 \\
\hline $\mathrm{Au}-22$ quilates & 63,45 & 46.80 \\
\hline
\end{tabular}

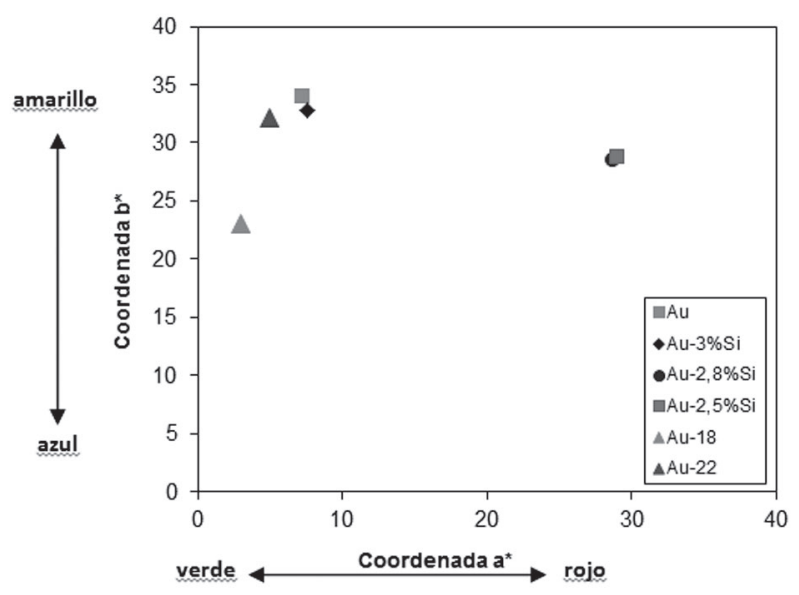

Figura 10. Coordenadas de color CIELab para las muestras de Au-Si comparadas con oro puro, y aleaciones de 18 y 22 quilates tradicionales.

Para la simulación como metal de aporte en soldadura de oro se muestran las imágenes de microscopía electrónica de barrido en la intercara sobre sustrato de Oro-Titanio en la figura 11. En las tres composiciones de Au-Si se verificó la formación del eutéctico Au-Si a partir del sustrato de oro, y la presencia de dendritas de oro, la estructura de solidificación es función de las variables empleadas para la soldadura. No se observa la formación de alguna fase intermetálica en la intercara entre el metal de aporte y el sustrato, y puede evidenciarse la solidificación del metal de aporte a partir del sustrato, infiriéndose, en forma cualitativa que se presenta buena mojabilidad, aunque en este estudio no fueron incluidos ensayos para la medición del ángulo. 

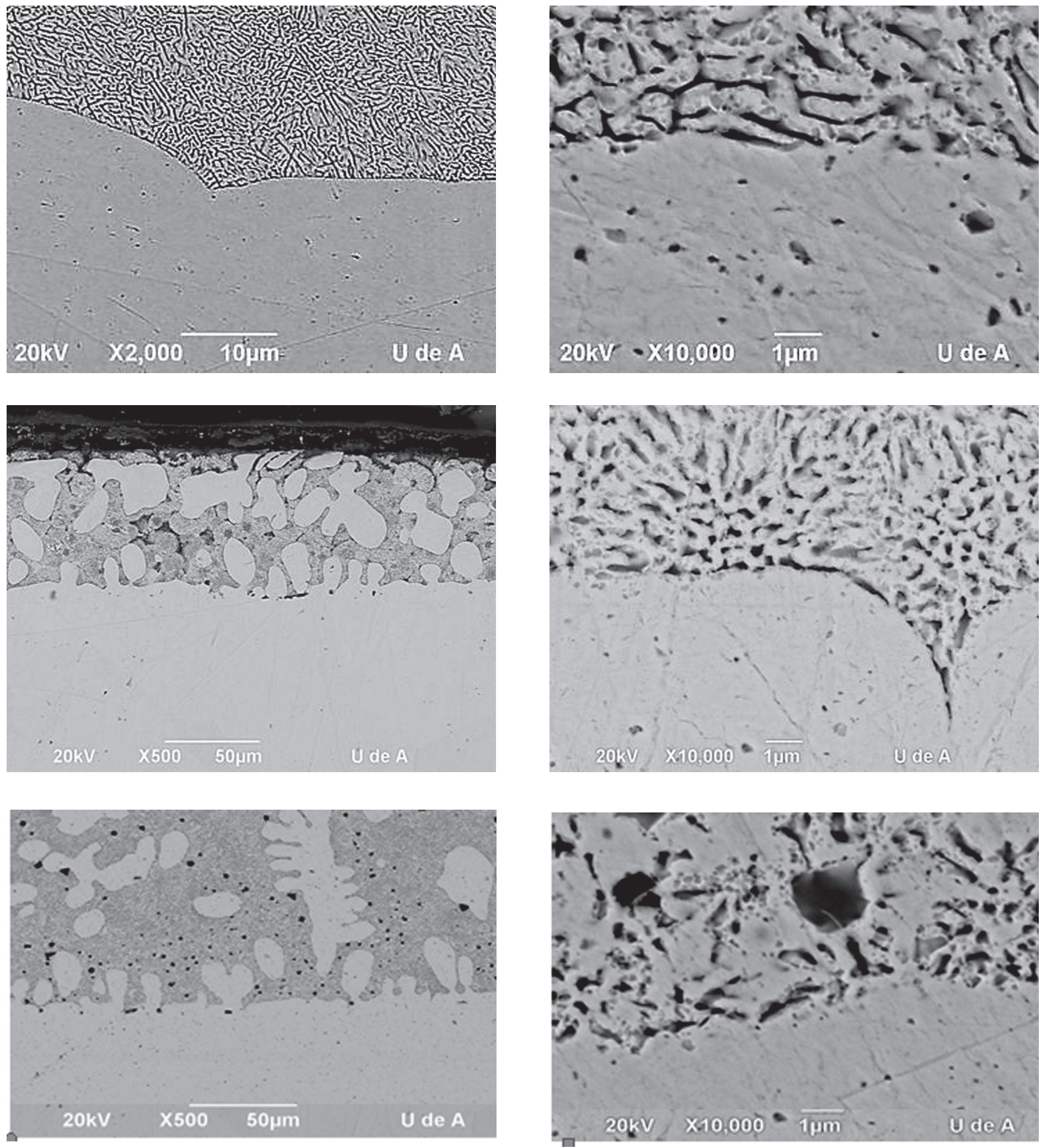

Figura 11. Micrografías de las uniones de Au-1\%Ti soldadas con propano con material de aporte $\mathrm{Au}-\mathrm{Si}$ en las proporciones a) $2,5 \% \mathrm{Si}$ b) $2,8 \% \mathrm{SI}$ y c) $3,0 \% \mathrm{Si}$ 


\section{Conclusiones}

Las aleaciones oro-silicio en composiciones cercanas al eutéctico entre el $2,5 \% \mathrm{Si}$ y $3,0 \% \mathrm{Si}$ han sido poco estudiadas y ofrecen una importante oportunidad de aplicación en los procesos de soldadura blanda, por su bajo punto de fusión, de $374^{\circ} \mathrm{C}$.

Los resultados de esta investigación revelan desviaciones de la aleación producida con respecto al comportamiento teórico esperado, por estar alejado de las condiciones de equilibrio, y con gran influencia de la velocidad de solidificación.

Los ensayos de dureza y microdurezas mostraron una tendencia importante en el endurecimiento de las aleaciones con el aumento del contenido de silicio, lo que resulta en un mejoramiento de propiedades mecánicas, con respecto al oro de alta pureza.

Los estudios de polarización, utilizando un medio de sudor artificial, mostraron que las aleaciones presentan una alta resistencia a la corrosión, muy similar al oro puro.

Los resultados del color de revelan que las aleaciones $\mathrm{Au}-$ -Si presentan valores similares a las aleaciones de 22 quilates tradicionales dado por los índices YI, que son en general el término de comparación de color más aplicado en la industria de la joyería.

\section{Agradecimientos}

Los autores agradecen el apoyo dado por el Fondo de Innovación Tecnológica de la Universidad de Antioquia, para la financiación de este trabajo.

\section{Referencias Bibliográficas}

[1] A.S. Mc Donald, G.H. Sistare. The metallurgy of some carat gold jewellery alloys. Gold Bulletin, 1978. p. 66-73.

[2] Gafner, G. "The development of 990 gold - titanium: its production, use and properties". Gold Bulletin. 22 (4), 1989.

[3] Sánchez H. Fabricación y caracterización de la aleación Au - 1,7\% Ti para aplicaciones odontológicas. Universidad de Antioquia Medellín, 2006

[4] Medina A. R. Fabricación Y Caracterización De Prototipos De Joyería En La Aleación Au -1,7\% Ti Obtenidos Por Microfusión A La Cera Perdida En Horno De Plasma. Universidad de Antioquia. Medellín. 2008.

[5] G, Humpston y D,M, Jacobson. Do 18 Carat Gold Solders Exist. Gold Bull.1994

[6] Sloboda, M.H. Industrial Gold Brazing Alloys: Their Present and Future Usefulness. Gold Bull. 1971; p 2-8

[7] Humpston, G.; Jacobson, D.M.; Sangha, S.P.S. Diffusion soldering: a New Low Temperature Process for Joining Carat Gold Jewellery. Gold Bull. 1993; p 90-104

[8] R.S Jackson. An improved 9 carat yellow gold casting alloy. Gold Bulletin, n 3, Vol 11. 1978. P 88-93.

[9] G. Gusmanoa, R. Montanarib, G. Montesperellic, M.E. Tatab, E. Bemporadd, M. Valente, S. Kaciulis, G. Mattogno. Influence of $\mathrm{Si}, \mathrm{Ni}$ and $\mathrm{Co}$ additions on gold alloy for investment cast process. Journal of Alloys and Compounds 325 (2001) 252-258.
[10] Alguacil, E.J. El Refino del Oro. Revista Metallurgia. Vol 31; No. 3 (1995); p. 182-191.

[11] Henderson, S. y Manchanda, D. White Gold Alloys: Colour Measurement and Grading. En: Gold Bulletin. Vol. 38; No. 2 (2005); p. 55-67. 